\title{
Towards Enhancing the Throughput and Eliminating the 4 Dimensions of Stitching Errors in Large Area, High Resolution SEM for Integrated Circuit Reverse Engineering and Connectomics
}

\author{
J. E. Sanabia ${ }^{1}$, C. E. Korman ${ }^{2}$, A. Popratiloff ${ }^{3}$, V. Boegli ${ }^{4}$, M. Rasche ${ }^{4}$, and R. Jede ${ }^{4}$ \\ ${ }^{1 .}$ International Applications Center, Raith America, Inc., Troy, NY, USA. \\ 2. Department of Electrical and Computer Engineering, The George Washington University, \\ Washington, DC, USA. \\ 3. GW Center for Nanofabrication and Imaging, The George Washington University, Washington, \\ DC, USA. \\ 4. Raith GmbH, Dortmund, Germany.
}

For large area, high resolution SEM imaging applications, such as integrated circuit (IC) reverse engineering, connectomics, block copolymer long-range ordering, and correlative microscopy, SEM instruments are inherently limited by small, uncalibrated fields of view (FOVs), imprecise sample positioning, and low electron detector bandwidths and efficiencies. These limitations affect image capture throughput, requiring more stage drive time, larger image overlaps, and a longer dwell time per pixel. Additionally, these instrument limitations introduce stitching errors in all 4 dimensions of the image data, X, Y, Z, and I (signal intensity). Throughput and stitching errors are cited challenges [1], and software alone cannot tenably correct stitching errors in large image datasets [2].

Although the CHIPSCANNER addresses some of these challenges by combining the resolution and flexibility of the SEM instrument with the accuracy (of the order $10 \mathrm{~nm}$ ) and stability of the electron beam lithography (EBL) instrument, still the CHIPSCANNER in its present form has its own limitations. The FOV size limit, electron detection bandwidth and efficiency, and sample contamination, damage, and charging effects can introduce stitching errors in all 4 dimensions and limit throughput. For the imaging of neuronal architecture (connectomics) with the CHIPSCANNER, reported last year at this conference [3], a $2.5 \mathrm{~mm}$ x $1.8 \mathrm{~mm}$ area cross section of mouse spinal cord took 24 hours to image at $10 \mathrm{~nm}$ x $10 \mathrm{~nm}$ pixel size, and had barely perceptible stitching boundaries due to discontinuities in the X, Y, and I dimensions (see Figure 1).

Reported here are new extremely large FOV, continuously moving laser interferometer stage, and electron detection technologies that together comprise a significant advance towards enhancing the throughput and eliminating the 4 dimensions of stitching errors in large area, high resolution SEM. Figure 2 shows the results from these technologies applied towards scanning a $1 \mathrm{~mm} \times 1 \mathrm{~mm}$ large area of a Chessy SEM calibration standard in less than one hour and with significantly reduced stitching errors. The continuously moving laser interferometer stage image capture strategy can virtually eliminate stitching errors in up to 3 dimensions, i.e. $\mathrm{X}$, Y, and I.

References:

[1] A A Wanner et al, Journal of Microscopy 259 (2015), pp. 137-142.

[2] K J Hayworth et al, Frontiers in Neural Circuits 8 (2014), pp. 1-18.

[3] C A Brantner et al, Proceedings of Microscopy \& Microanalysis (2015) pp. 921-922. 


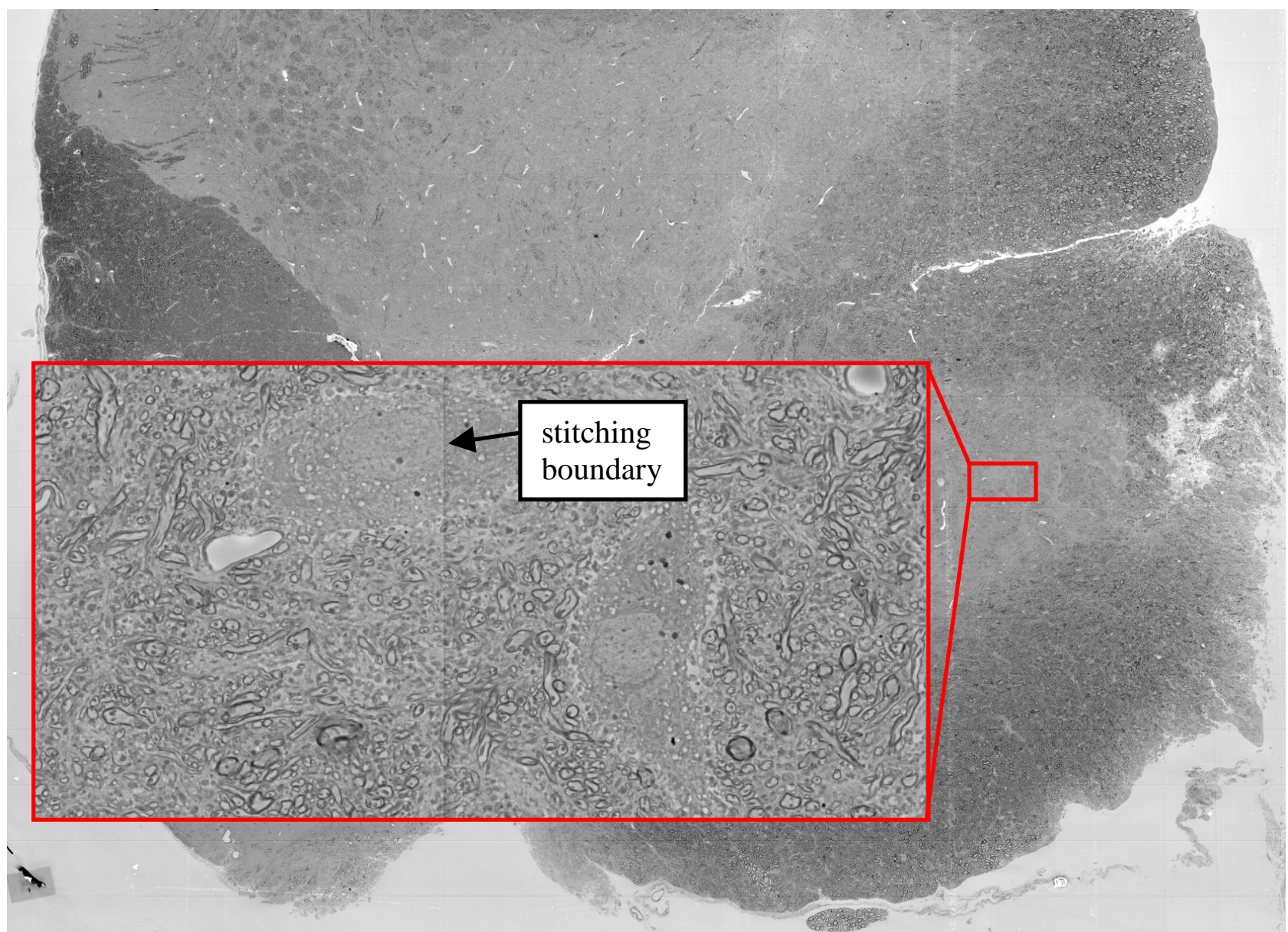

Figure 1. A $2.5 \times 1.8 \mathrm{~mm}$ area of spinal cord cross section captured over the course of 24 hours [3], with a $0.1 \times 0.2 \mathrm{~mm}$ section extracted and displayed at higher resolution.

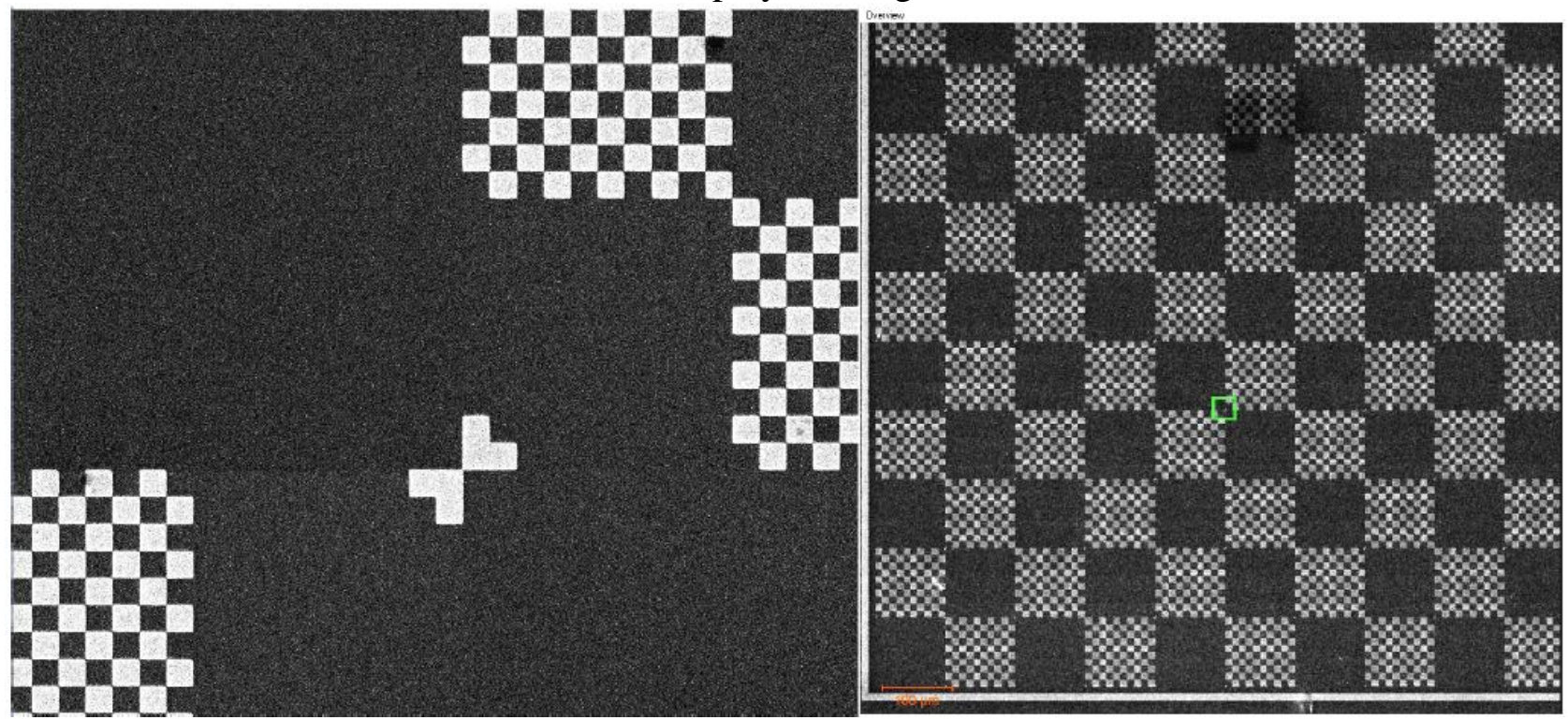

Figure 2. New extremely large FOV, continuously moving laser interferometer stage, and electron detection technologies result in scanning a $1 \mathrm{~mm}$ x $1 \mathrm{~mm}$ large area of a Chessy SEM calibration standard in less than one hour and with significantly reduced stitching errors. 